\title{
HOW QCD EVOLUTION KERNELS DEPEND ON THE TYPE OF EVOLUTION VARIABLE*
}

\author{
S. JAdAch ${ }^{\mathrm{a}}$, A. Kusina ${ }^{\mathrm{a}}$, W. PŁaczeK ${ }^{\mathrm{b}}$, M. SkrzyPeK ${ }^{\mathrm{a}}$ \\ ${ }^{a}$ H. Niewodniczański Institute of Nuclear Physics, Polish Academy of Sciences \\ Radzikowskiego 152, 31-342 Kraków, Poland \\ ${ }^{\mathrm{b}} \mathrm{M}$. Smoluchowski Institute of Physics, Jagiellonian University \\ Łojasiewicza 11, 30-348 Kraków, Poland
}

(Received October 30, 2017)

We show that by changing the upper phase space limit in calculation of an evolution kernel, one can change its functional form. This happens already at the NLO level, e.g. when the upper phase space limit is defined in terms of maximum of transverse momenta. The upper phase space limit of the evolution kernel corresponds to the evolution variable used in a Parton Shower, and this dependence means that the different kernels need to be used depending on the ordering of the Parton Shower.

DOI:10.5506/APhysPolB.48.2275

\section{Introduction}

Parton Showers (PS) [1-3] are fundamental tools in the data analysis. They are based on factorization theorems $[4,5]$ and QCD evolution equations [6]. To date, all PS are of the improved Leading Order (LO) type. The project of constructing PS based on Next-to-Leading Order (NLO) evolution has been pursued by the Kraków group from IFJ PAN ${ }^{1}$ [7-10] as well as other groups [11-13]. The evolution kernel is an essential element of the factorization program. Let us present it on the example of the $Z$-boson production. In Fig. 1, we present this process with a complete set of Initial State Radiation (ISR) corrections. The first step in kernel construction is the reorganization of the scattering in terms of the ladders of two-particleirreducible objects, denoted $K_{0}$, and the hard process, labeled as $C_{0}$. These objects contain all Feynman graphs that cannot be divided by cutting two quark lines on the sides of the ladder. The crucial step in kernel construction is the inclusion of projection operators, as shown in Fig. 2.

* Presented by M. Skrzypek at the XLI International Conference of Theoretical Physics "Matter to the Deepest", Podlesice, Poland, September 3-8, 2017.

${ }^{1}$ H. Niewodniczański Institute of Nuclear Physics, Polish Academy of Sciences. 


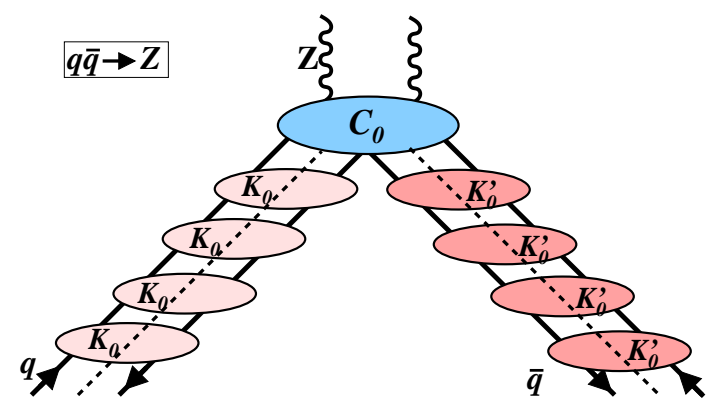

Fig. 1. The ladders of two-particle-irreducible sets $K_{0}$ in the Drell-Yan-type process.

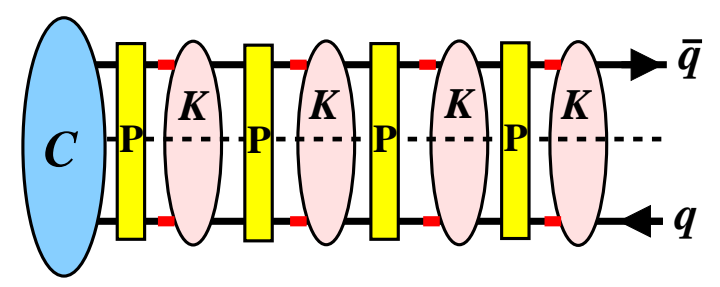

Fig. 2. The ladder split with the help of the projection operators $P$.

The action of this operator consists of the following steps:

1. It closes fermionic lines with the projector $\not h / 4 n q$ and the matrix $\not p$

$$
\Gamma_{q q}^{\text {unint }}=\operatorname{Tr}\left[\frac{\not h}{4 n q} K \not p\right]
$$

where $p$ and $q$ are correspondingly the incoming and outgoing momenta, and $n$ is a time-like vector defining axial gauge.

2. It puts incoming parton on-shell, $p^{2}=0$.

3. It extracts pole part (PP) of the expression, ensuring the $\overline{\mathrm{MS}}$ scheme.

4. It sets upper integration limit $Q^{2}$ (cut-off) on the evolution variable.

Altogether, the action of the projection operator results in

$$
\Gamma_{q q}=x \operatorname{PP} \int^{Q^{2}} \mathrm{dLips}^{(m)} \delta\left(x-\frac{q n}{p n}\right) \Gamma_{q q}^{\mathrm{unint}} .
$$

We do not specify here the integration/evolution variable. In the original paper [5], it is the virtuality $-q^{2}<Q^{2}$. In this note, we will analyze a 
number of other choices as this is the point of our interest. The kernel $P$ is then defined as the residue of $\Gamma$ :

$$
\begin{aligned}
\Gamma_{q q} & =\delta_{1-x}+\frac{1}{\epsilon}\left[\left(\frac{\alpha}{2 \pi}\right) P^{(1)}+\frac{1}{2}\left(\frac{\alpha}{2 \pi}\right)^{2} P^{(2)}+\ldots\right]+\ldots, \\
P_{q q} & =\left(\frac{\alpha}{2 \pi}\right) P^{(1)}+\left(\frac{\alpha}{2 \pi}\right)^{2} P^{(2)}+\ldots
\end{aligned}
$$

\section{Technical details}

We will analyze the $P_{q q}$ kernel and consider three groups of different cutoffs (ordering variables): (1) the standard one of Ref. [5], i.e. the virtuality, $-q^{2}<Q^{2} ;(2)$ related to the transverse momentum of the emitted partons, $\max \left\{k_{1 \perp}, k_{2 \perp}\right\}<Q$ or $k_{1 \perp}+k_{2 \perp}<Q ;(3)$ related to the rapidity (angle) of the emitted partons, $\max \left\{k_{1 \perp} / \alpha_{1}, k_{2 \perp} / \alpha_{2}\right\}<Q$ or $\left|k_{1 \perp}+k_{2 \perp}\right| /\left(\alpha_{1}+\alpha_{2}\right)<Q$. The variables $\alpha$ and $\beta$ are the Sudakov variables defined in the usual way $k_{i}=\alpha_{i} p+\beta_{i} n+k_{i \perp}^{(m)}$. As a consequence, we have the standard relation $k_{\perp}^{2}=2 p n \alpha \beta$, hence the rapidity-like variable is $k_{\perp} / \alpha \sim \sqrt{\beta / \alpha}$, where we defined $k_{i \perp} \equiv\left|\vec{k}_{i \perp}\right|$.

The graphs that contribute can be classified by the power of $\epsilon$-poles: $1 / \epsilon, 1 / \epsilon^{2}$ and $1 / \epsilon^{3}$. We will discuss them in turns. Here, we summarize the results. The single-pole graphs are invariant with respect to the change of the integration limit. The double-pole graphs exhibit two mechanisms of cancellation: between the graph and its counter-term, leading to the invariance, and between the real and virtual ones, leading to the change of the kernel. The triple-pole graph is also invariant provided one modifies the regularization prescription of spurious singularities [14]. The double-pole graphs are shown in Fig. 3 and the triple-pole one is presented in Fig. 4.
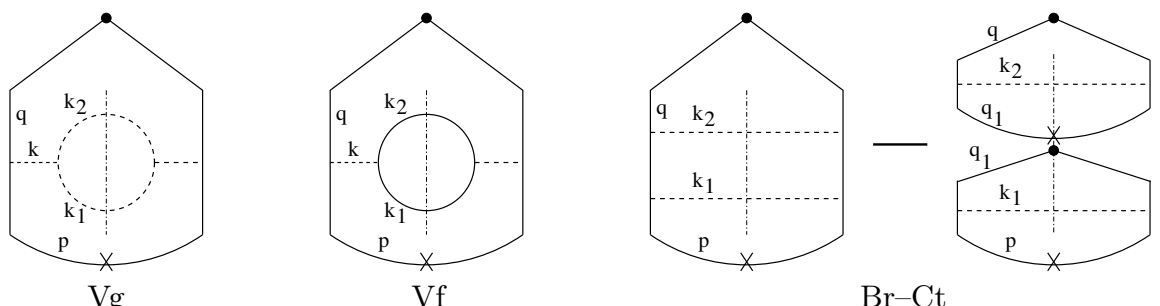

$\mathrm{Br}-\mathrm{Ct}$

Fig. 3. The real graphs with double poles contributing to the NLO non-singlet $P_{q q}$ kernel. The dashed lines represent gluons.

A common feature of all the graphs is the presence of the singularity in the virtuality variable $q^{2}=0$. This collinear singularity can, however, be eliminated if, instead of calculating the entire contribution of a given graph, 


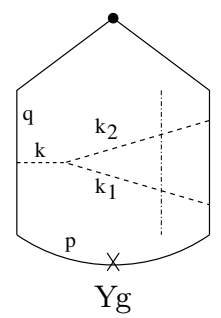

Fig. 4. The real graph with triple pole contributing to the NLO non-singlet $P_{q q}$ kernel. The dashed lines represent gluons.

one calculates only the difference between the standard virtuality cut-off $\left(-q^{2}<Q^{2}\right)$ and the new cut-off

$$
\Delta \Gamma^{X-q^{2}}=\Gamma\left(X<Q^{2}\right)-\Gamma\left(-q^{2}<Q^{2}\right),
$$

where $X$ stands for the new cut-off. This way the singular region around $q^{2}=0$ is excluded. Keeping in mind that the kernel is proportional to the coefficient of the $1 / \epsilon$ term, as a consequence, the single pole graphs will not contribute at all to this difference. As for the double pole graphs, one can identify the other integration variable which is singular and expand in it with the help of the standard formula

$$
\mathrm{d} k^{2}\left(k^{2}\right)^{-1+\epsilon} \rightarrow \mathrm{d} k^{2} \frac{1}{\epsilon} \delta\left(k^{2}\right) .
$$

Next, one can set $\epsilon$ to 0 in all other places.

In the case of the Br-Ct graphs (see Fig. 3), one uses in Eq. (6) the $k_{1 \perp}^{2}$ instead of $k^{2}$ and one can show that the counter-term compensates exactly the change in the bremsstrahlung graph and there is no change in the kernel.

The situation looks differently in the case of Vf and Vg graphs (see Fig. 3). Here, the leftover singularity is located directly in the $k^{2}$ variable. We show here only the initial formula based on the Feynman graphs (for the Vg graph):

$$
\begin{aligned}
\Gamma_{G}= & \frac{1}{2} C_{G} C_{\mathrm{F}} g^{4} x \operatorname{PP}\left[\frac{1}{\mu^{4 \epsilon}} \int \mathrm{d} \Psi \delta\left(x-\frac{q n}{p n}\right) \frac{1}{q^{4}} W_{G}\right] \\
\mathrm{d} \Psi= & \frac{\mathrm{d}^{m} k_{1}}{(2 \pi)^{m}} 2 \pi \delta^{+}\left(k_{1}^{2}\right) \frac{\mathrm{d}^{m} k_{2}}{(2 \pi)^{m}} 2 \pi \delta^{+}\left(k_{2}^{2}\right) \\
= & (2 \pi)^{-2 m+2} \frac{1}{4} \frac{\mathrm{d} \alpha_{1}}{\alpha_{1}} \frac{\mathrm{d} \alpha_{2}}{\alpha_{2}} \mathrm{~d}^{m-2} \vec{k}_{1 \perp} \mathrm{d}^{m-2} \vec{k}_{2 \perp}, \\
W_{G}= & \frac{1}{4 q n} \frac{1}{k^{4}} \operatorname{Tr}\left(\hat{n} \hat{q} \gamma^{\mu} \hat{p} \gamma^{\lambda} \hat{q}\right) d_{\nu^{\prime \prime} \nu^{\prime}}\left(k_{2}\right) d_{\mu \mu^{\prime \prime}}\left(k_{1}+k_{2}\right) d_{\lambda^{\prime \prime} \mu^{\prime}}\left(k_{1}\right) d_{\mu^{\prime} \lambda}\left(k_{1}+k_{2}\right) \\
& \times V\left(k_{1}^{\mu^{\prime \prime}}+k_{2}^{\mu^{\prime \prime}},-k_{2}^{\nu^{\prime \prime}},-k_{1}^{\lambda^{\prime \prime}}\right) V\left(k_{1}^{\mu^{\prime}}, k_{2}^{\nu^{\prime}},-k_{1}^{\lambda^{\prime}}-k_{2}^{\lambda^{\prime}}\right) .
\end{aligned}
$$


In this case, it is convenient to change variables in the transverse plane, so that the singular variables $k^{2}$ and $q^{2}$ become diagonal

$$
\begin{aligned}
\vec{\kappa}_{1} & =\frac{\alpha_{1}}{\alpha_{1}+\alpha_{2}}\left(\vec{k}_{1 \perp}+\vec{k}_{2 \perp}\right), & \vec{\kappa}_{2} & =\frac{\alpha_{1} \alpha_{2}}{\alpha_{1}+\alpha_{2}}\left(\frac{\vec{k}_{2 \perp}}{\alpha_{2}}-\frac{\vec{k}_{1 \perp}}{\alpha_{1}}\right), \\
k^{2} & =\frac{(1-x)^{2}}{\alpha_{1} \alpha_{2}} \kappa_{2}^{2}, & -q^{2} & =\frac{1-x}{\alpha_{1}}\left(\kappa_{1}^{2} \frac{1}{\alpha_{1}}+\kappa_{2}^{2} \frac{x}{\alpha_{2}}\right) .
\end{aligned}
$$

Further details of calculation can be found in Ref. [15]. In the following, we present only the final results.

\section{Results}

The results depend on the choice of the cut-off. As the first choice, let us present cut-off on the scalar sum of the transverse momenta, $k_{1 \perp}+k_{2 \perp}<Q$. Added together, the real emission graphs Vf and Vg give

$$
\begin{aligned}
\Delta \Gamma_{\mathrm{Vf}+\mathrm{Vg}}^{k_{\perp}-q} & =C_{\mathrm{F}}\left(\frac{\alpha_{\mathrm{S}}}{2 \pi}\right)^{2} \frac{1}{2 \epsilon} \frac{1+x^{2}}{1-x} \ln \frac{1}{1-x}\left[-\beta_{0}+4 C_{\mathrm{A}}\left(I_{0}+\ln (1-x)\right)\right] \\
\beta_{0} & =\frac{11}{3} C_{\mathrm{A}}-\frac{4}{3} T_{\mathrm{F}} .
\end{aligned}
$$

The virtual partners of these graphs are also modified by the cut-off change. The modification is however simple, and we obtain just the opposite to the real correction

$$
\Delta \Gamma_{\text {virtual }}^{k_{\perp}-q}=-\Delta \Gamma_{\mathrm{Vf}+\mathrm{Vg}}^{k_{\perp}-q} .
$$

Having added real and virtual corrections, we get complete cancellation the kernel remains unchanged. The same invariance of the kernel holds for the rapidity (angular) variables $\max \left\{k_{1 \perp} / \alpha_{1}, k_{2 \perp} / \alpha_{2}\right\}<Q$ and $\mid \vec{k}_{1 \perp}+$ $\vec{k}_{2 \perp} \mid /\left(\alpha_{1}+\alpha_{2}\right)<Q$.

The situation becomes interesting for the most natural $k_{\perp}$-type cut-off — the one on the maximum of $k_{\perp}$-s, i.e. $\max \left\{k_{1 \perp}, k_{2 \perp}\right\}<Q$. The result for Vf plus Vg real graphs is the following:

$$
\begin{aligned}
\Delta \Gamma_{\mathrm{Vf}+\mathrm{Vg}}^{\max k_{\perp}-q}= & \Delta \Gamma_{\mathrm{Vf}+\mathrm{Vg}}^{k_{\perp}-q} \\
& +C_{\mathrm{F}}\left(\frac{\alpha_{\mathrm{S}}}{2 \pi}\right)^{2} \frac{1}{\epsilon} \frac{1+x^{2}}{1-x}\left[C_{\mathrm{A}}\left(\frac{\pi^{2}}{3}-\frac{1}{8}\right)+\beta_{0}\left(\ln 2-\frac{23}{24}\right)\right] .
\end{aligned}
$$

As compared to the previous case of Eq. (10), we have now an additional term. The virtual contribution has only one real parton, and the cut-off is 
identical in both cases. Therefore, it remains unchanged as compared to the previous case of Eq. (11). This leads to the modification of the kernel

$$
\begin{aligned}
& P_{q q}\left(\max \left\{k_{1 \perp}, k_{2 \perp}\right\}<Q\right)-P_{q q}\left(-q^{2}<Q^{2}\right) \\
& =C_{\mathrm{F}}\left(\frac{\alpha_{\mathrm{S}}}{2 \pi}\right)^{2} \frac{1+x^{2}}{1-x}\left[C_{\mathrm{A}}\left(\frac{2 \pi^{2}}{3}-\frac{1}{4}\right)+\beta_{0}\left(2 \ln 2-\frac{23}{12}\right)\right] .
\end{aligned}
$$

Equation (13) is the principal new result of the presented work. It demonstrates that there are evolution variables for which kernels have a different form.

We conclude this section by formulating a general method of analyzing various cut-offs in the calculation of the Yg graph. In Fig. 5, we show the $\kappa_{1}$ and $\kappa_{2}$ plane. The singularities lie at the origin of the frame $\left(q^{2}=0\right)$ and along the line $\kappa_{2}^{2} \sim k^{2}=0$. The integration path, defined by the $\delta\left(k^{2}\right)$ of Eq. (6) is marked by the thick black line along $\kappa_{1}$ axis between the crossing points of the axis with $-q^{2}=Q_{0}^{2}$ and the cut-off. The cut-offs are the light gray/blue and thick gray/green line. It is evident that all cut-offs crossing $\kappa_{1}$ in the same point lead to the same result.

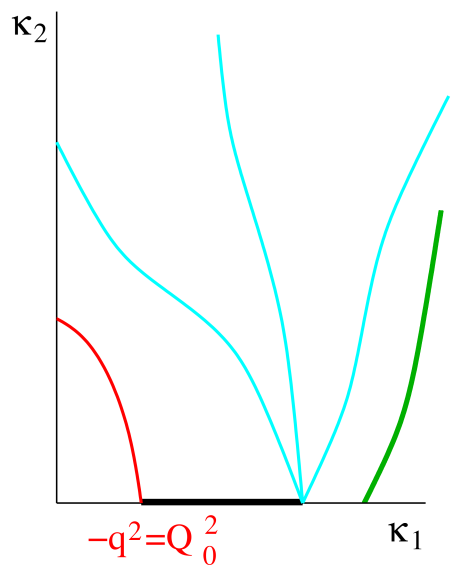

Fig. 5. The $\left(\kappa_{1}, \kappa_{2}\right)$ plane. A family of equivalent cut-off lines is shown in light gray/blue. Thick gray/green line shows a non-equivalent cut-off. At the bottom left, the $-q^{2}=Q_{0}^{2}$ cut-off is plotted in thin black/red. The integration path is marked by the thick black line along $\kappa_{2}=0$.

\section{Graph with triple poles}

When calculated in a traditional way [5], the graph Yg of Fig. 4 contains $1 / \epsilon^{3}$ poles along with the $\ln \delta$ singularities [16]. The $\delta$ is a small dummy regulator introduced into the calculation already at the level of the Feynman 
rules in the form of the PV prescription (see Ref. [5]). Namely, the gluon propagator in the axial gauge has the form of

$$
\frac{1}{l^{2}}\left(g^{\mu \nu}-\frac{l^{\mu} n^{\nu}+n^{\mu} l^{\nu}}{n l}\right), \quad \frac{1}{n l} \rightarrow \frac{1}{[n l]_{\mathrm{PV}}}=\frac{n l}{n l^{2}+\delta^{2} n p^{2}}
$$

with the spurious singularity $1 / n l$. It cancels in the final result, nonetheless an intermediate regularization is needed, and the PV prescription has been chosen for this purpose. In Refs. [14, 17], it has been noticed that the above PV prescription can be applied not only to the gluon propagator, but also to all the remaining singularities in the light-cone plus variable

$$
\frac{\mathrm{d}^{m} l}{l_{+}^{1-\epsilon}} \rightarrow \mathrm{d}^{m} l\left[\frac{1}{l_{+}}\right]_{\mathrm{PV}}\left(1+\epsilon \ln l_{+}+\epsilon^{2} \frac{1}{2} \ln ^{2} l_{+}+\ldots\right), \quad l_{+}=\frac{n l}{n p} .
$$

This prescription, named "New PV" (NPV), leads to the same NLO kernels, but replaces the higher-order epsilon poles by the $\ln \delta$ terms in individual contributions to the kernels. In particular, in the contribution of the real $\mathrm{Yg}$ graph discussed here, in the NPV prescription, the $1 / \epsilon^{3}$ poles are reduced to the single ones (at the expense of the $\ln ^{2} \delta$ terms). Hence, as a single pole graph, Yg will not contribute anymore to the difference defined in Eq. (5).

\section{Summary and conclusions}

Let us summarize the results of this work. An obvious remark is that the LO kernels are insensitive to the change of the evolution variable. The dependence appears at the NLO level and only for selected graphs and selected cut-offs. Specifically, it exhibits only for the real diagram with FSR-type gluonstrahlung ( $\mathrm{Vg}$ and $\mathrm{Vf}$ ) and for the upper integration limit $\max \left\{k_{1 \mathrm{~T}}, k_{2 \mathrm{~T}}\right\} \leq Q$. The other double-pole graphs (of the ISR gluonstrahlung type) as well as the single pole graphs do not contribute. Also, the other cut-offs, like $\left|\vec{k}_{1 \mathrm{~T}}+\vec{k}_{2 \mathrm{~T}}\right| \leq Q, k_{1 \mathrm{~T}}+k_{2 \mathrm{~T}} \leq Q$ and rapidities, do not change the kernel.

The effect can be attributed to the mismatch between the kinematic limit in the real and virtual graphs. From the point of view of the classical paper of Ref. [5], the dependence of the kernel on the upper integration limit is not acceptable as it contradicts the theorem proven therein. One may ask therefore whether this effect could be a result of the, somewhat ad $h o c$, introduction of the PV regularization in the CFP scheme, or the New PV regularization used in this work. It is also possible that the source is more fundamental - the axial gauge itself. On the other hand, there is a more natural explanation, that this is just a different factorization scheme and the change will be compensated by the appropriate change of the hard process. 


\section{REFERENCES}

[1] T. Sjöstrand et al., Comput. Phys. Commun. 191, 159 (2015) [arXiv: 1410.3012 [hep-ph]].

[2] J. Bellm et al., Eur. Phys. J. C 76, 196 (2016) [arXiv:1512.01178 [hep-ph]].

[3] T. Gleisberg et al., J. High Energy Phys. 9002, 007 (2009) [arXiv:0811.4622 [hep-ph]].

[4] R.K. Ellis et al., Phys. Lett. B 78, 281 (1978).

[5] G. Curci, W. Furmanski, R. Petronzio, Nucl. Phys. B 175, 27 (1980).

[6] L.N. Lipatov, Sov. J. Nucl. Phys. 20, 95 (1975); V.N. Gribov, L.N. Lipatov, Sov. J. Nucl. Phys. 15, 438 (1972); G. Altarelli, G. Parisi, Nucl. Phys. B 126, 298 (1977); Yu.L. Dokshitzer, Sov. Phys. JETP 46, 64 (1977).

[7] S. Jadach, M. Skrzypek, Acta Phys. Pol. B 40, 2071 (2009) [arXiv:0905.1399 [hep-ph]].

[8] S. Jadach, M. Skrzypek, A. Kusina, M. Sławińska, PoS RADCOR2009, 069 (2010) [arXiv:1002.0010 [hep-ph]].

[9] S. Jadach, A. Kusina, M. Skrzypek, M. Slawinska, Nucl. Phys. Proc. Suppl. 205-206, 295 (2010) [arXiv:1007.2437 [hep-ph]].

[10] S. Jadach, A. Kusina, W. Płaczek, M. Skrzypek, Acta Phys. Pol. B 44, 2179 (2013) [arXiv:1310.6090 [hep-ph]].

[11] S. Alioli et al., J. High Energy Phys. 1309, 120 (2013) [arXiv: 1211.7049 [hep-ph]].

[12] K. Hamilton, P. Nason, G. Zanderighi, J. High Energy Phys. 1210, 155 (2012) [arXiv:1206.3572 [hep-ph]].

[13] Z. Nagy, D.E. Soper, arXiv:1705.08093 [hep-ph].

[14] O. Gituliar, S. Jadach, A. Kusina, M. Skrzypek, Phys. Lett. B 732, 218 (2014) [arXiv: 1401.5087 [hep-ph]].

[15] S. Jadach, A. Kusina, W. Płaczek, M. Skrzypek, J. High Energy Phys. 1608, 092 (2016) [arXiv: 1606.01238 [hep-ph]].

[16] G. Heinrich, Improved Techniques to Calculate Two-loop Anomalous Dimensions in QCD, Ph.D. Thesis, Swiss Federal Institute of Technology, Zurich, 1998, http://dx.doi.org/10.3929/ethz-a-001935934

[17] M. Skrzypek, O. Gituliar, S. Jadach, A. Kusina, PoS LL2014, 059 (2014) [arXiv: 1407.6261 [hep-ph]]. 